\title{
Extension of Maier-Meier Model on Cholesteric Liquid Crystals
}

\author{
J. HOFFMANN \\ Institute of Molecular Physics, Polish Academy of Sciences \\ Smoluchowskiego 17, 60-179 Poznań, Poland
}

(Received March 16, 2004)

\begin{abstract}
Based on the Maier-Meier theory, a model is proposed in which the electric permittivity values of the cholesteric liquid crystals can be linked to their molecular parameters $(\mu-$ dipole moment, $\alpha$-polarizability). To accomplish this project there were defined the transformation principles from the liquid crystal structure frame to the molecular frame, and a method for rendering the single molecule parameters $(\mu, \alpha)$ into a set of averaged quantities of the macroscopic ensemble was offered. This model was used to calculate a dipole moment and polarizability for two liquid crystalline materials from electric permittivity measurements. The results are compared with the data obtained from MP3 calculations.
\end{abstract}

PACS numbers: $61.30 . \mathrm{Eb}$

\section{Introduction}

The cholesteric liquid crystals (cholesterics) also known as chiral nematics are characterized by spiral symmetry axis (a helix) formed in their volume [1]. One could believe that the dielectric properties of the cholesterics are predictable on the basis of the physical parameters of a single molecule constituting the liquid crystal. It should imply a step forward in discussing the physical properties of this group of materials. Starting from the description in terms of mesophase this step would enable us to attain the level molecule. Three particular problems must be clarified before such model is effected:

1. Orientational order of the molecules of the liquid crystal.

2. Definition of the local field.

3. Relation between "macroscopic parameters" $(\varepsilon, n)$ and "molecular parameters" $(\mu, \alpha)$. 
For nematic liquid crystals these problems have been solved by Maier and Meier [2]. These authors have made an extension of the Onsager theory [3]. In their theory a molecule is represented by an anisotropic polarizability $\alpha$ with principal elements $\alpha_{\|}$and $\alpha_{\perp}$ in spherical cavity of radius $a$. Furthermore, there is a permanent dipole moment $\mu$ that makes an angle $\beta$ with the direction $\alpha_{\|}$(direction of long axis of elliptic molecule). This calculation involves the following approximation: the anisotropy of the electric permittivity is not taken into account for calculations of the cavity factor, reaction field factor, and the internal field factor. This approximation implies that internal electric field is taken to be isotropic, just as in the case of the Vuks equation for nonpolar molecules [4].

Maier and Meier equations account satisfactorily for many essential features of permittivity of nematic liquid crystals with polar molecules and are still used for calculations of the molecular parameters from electric permittivity measurements (see for example [5]).

An extension of the Maier and Meier theory to the cholesteric liquid crystals will be attempted in this study.

\section{Experiment}

The measurements described in this study concern the cholesteric phase of two liquid crystal materials. First of them, [4-(2-methylbutyl) phenyl 4'-cyano1,1'-biphenyl-4carboxylate] shortly called as D4, is produced at laboratory of Professor R. Dąbrowski.

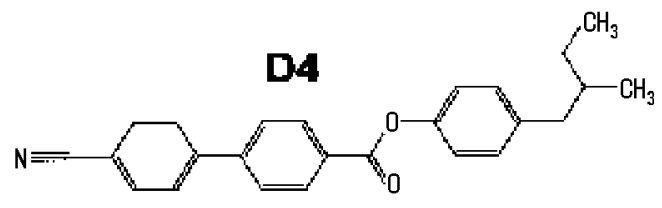

The other one, known as CE8, ([4-(2-methylbutyl) phenyl 4'-octyl-1,1'-biphenyl-4carboxylate] is supplied by $\mathrm{BDH}$ :

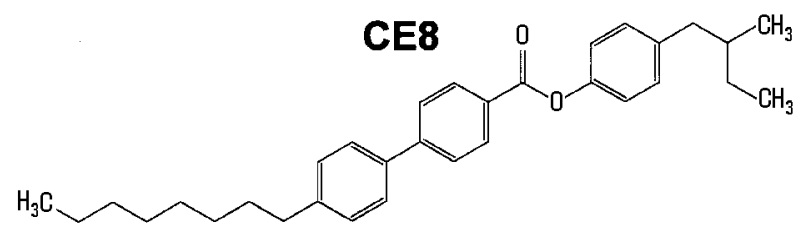

Two measuring configurations are available: the finger print texture, when the helix axis $\boldsymbol{L}$ lies in the plane parallel to the electrodes and the Grandjean texture when the helix axis $\boldsymbol{L}$ is perpendicular to the surface of the sample. It means that, with respect to the laboratory reference system, the measurements are made either in the direction parallel or perpendicular to axis of the helix. 
Electric permittivity was measured using cells producing uniform alignment of the samples of $25 \mu \mathrm{m}$ thickness. The measurements were made using Hewlett Packard Impedance Analyzer 4192A. Temperature was stabilized in a Mettler microscopic hot stage and Unipan $650 \mathrm{H}$ temperature controller. Thermal stability of $\pm 0.01 \mathrm{~K}$ could be easily maintained. Birefringence of the samples was measured with the modified wedge method. The lens of curvature radius $21.7 \mathrm{~mm}$ was employed. The interference pattern was obtained at $\lambda=590 \mathrm{~nm}$ monochromatic light and the distances between dark fringes measured using the calibrated eyepiece of the microscope. The optical measurements were performed using the same temperature control.

\section{Theory}

Before attempting the discussion of dielectric properties of the cholesterics it would be worthwhile summarizing the specific features of this kind of a structure. The anisotropic molecules of which a liquid crystal is composed exhibit orientational order. Direction of the local optical axis is shown with the unit vector $n-$ the director. Its direction changes smoothly in the volume of the liquid crystal as described by the equations of helix ordering of a pitch $p$. Locally, i.e. in the volumes thinner than the pitch: $d \ll p$, the orderings of cholesterics and the nematics are indistinguishable. On these principles, the application of the procedure used in the outlining the model of the dielectric properties of the nematics to the cholesterics system seems well justified.

The ordering of the liquid crystal cholesteric phase is characterized by the occurrence of the spiral symmetry axis $\boldsymbol{L}$ perpendicular to the director $\boldsymbol{n}$. With the electric permittivity measured at the two available experimental configurations the determination of the permittivity tensor $\varepsilon_{i j}^{\mathrm{h}}$ becomes possible. In the case of the finger print texture, in which the electric field is perpendicular to the direction of the helix axis $(\boldsymbol{E} \perp \boldsymbol{L}) \varepsilon_{\perp}^{\mathrm{h}}$ is measured. The parallel component $\varepsilon_{\|}^{\mathrm{h}}$ of the electric permittivity $\varepsilon_{i j}^{\text {h }}$ is obtained from the measurements for the samples exhibiting the Grandjean texture i.e. $\boldsymbol{E} \| \boldsymbol{L}$. In the main axis frame defined with respect to the helix axis $\left(\boldsymbol{L} \| \boldsymbol{X}_{3}\right)$ the electric permittivity tensor $\varepsilon_{i j}^{\mathrm{h}}$ is of the form

$$
\varepsilon_{i j}^{\mathrm{h}}=\left(\begin{array}{ccc}
\varepsilon_{\perp}^{\mathrm{h}} & 0 & 0 \\
0 & \varepsilon_{\perp}^{\mathrm{h}} & 0 \\
0 & 0 & \varepsilon_{\|}^{\mathrm{h}}
\end{array}\right) .
$$

The tensor $\varepsilon_{i j}^{\mathrm{h}}$ can be transformed to the coordinates system fixed with the director $\boldsymbol{n}$ frame. The transformation principles have been discussed previously [6].

The relations between the components of the permittivity tensor $\varepsilon_{i j}$ (expressed in the $\boldsymbol{n}$ vector coordinates system) and $\varepsilon_{i j}^{\mathrm{h}}$ are following [6]:

$$
\varepsilon_{\|}^{h}=\varepsilon_{\perp},
$$




$$
\varepsilon_{\perp}^{\mathrm{h}}=\frac{1}{2}\left(\varepsilon_{\|}+\varepsilon_{\perp}\right) .
$$

$\varepsilon_{\|}$and $\varepsilon_{\perp}$ are the elements of the electric permittivity tensor $\varepsilon_{i j}$. In the main axis representation so chosen that the $\boldsymbol{X}_{3}$ is aligned parallel to the director (nematic type order), i.e. $n \| \boldsymbol{X}_{3}$, the tensor $\varepsilon_{i j}$ can be written

$$
\varepsilon_{i j}=\left(\begin{array}{ccc}
\varepsilon_{\perp} & 0 & 0 \\
0 & \varepsilon_{\perp} & 0 \\
0 & 0 & \varepsilon_{\|}
\end{array}\right) .
$$

Theory of Maier and Meier [2] derived the equations, which correlate dielectric properties of nematic liquid crystals to molecular parameters. In this study transformation rules from nematic to cholesteric order were used to complete procedure described by these authors.

Hence the additional assumptions will be made:

- Locally, i.e. at the depth $d$ less than the helix pitch $(d \ll p)$ the distribution of the molecular symmetry axes is governed by the distribution function defining the order parameter $S$, as it is in the nematics.

To calculate cavity factor $h$, reaction field factor $f$ and internal field factor $F$ the following mean values are used:

$$
\begin{aligned}
& \left.\begin{array}{rl}
\bar{\varepsilon} & =\frac{\varepsilon_{\|}+2 \varepsilon_{\perp}}{3} \\
\bar{\alpha} & =\frac{\alpha_{\|}+2 \alpha_{\perp}}{3}
\end{array}\right\} \text { mean values of the electric permittivity and polarizability, } \\
& f=\frac{\bar{\varepsilon}-1}{2 \pi \varepsilon_{0} a^{3}(2 \bar{\varepsilon}+1)}, \quad F=\frac{1}{1-f \bar{\alpha}}, \quad h=\frac{3 \bar{\varepsilon}}{2 \bar{\varepsilon}+1} .
\end{aligned}
$$

- Components of the polarizability tensor of cholesteric liquid crystal are given by equations

$$
\left\langle\alpha_{\|}\right\rangle^{\mathrm{h}}=N\left(\bar{\alpha}-\frac{1}{3} \Delta \alpha S\right), \quad\left\langle\alpha_{\perp}\right\rangle^{\mathrm{h}}=N\left(\bar{\alpha}+\frac{1}{6} \Delta \alpha S\right)
$$

( $N$ - number of molecules, $\Delta \alpha=\alpha_{\|}-\alpha_{\perp}$ ).

- Orientational polarization along and perpendicular to the $\boldsymbol{L}$ (helix axis) are given by

$$
\begin{aligned}
\left\langle\mu_{\|}^{2}\right\rangle^{\mathrm{h}} & =\frac{1}{3} \mu^{2}\left[1+\frac{1}{2}\left(1-3 \cos ^{2} \beta\right) S\right], \\
\left\langle\mu_{\perp}^{2}\right\rangle^{\mathrm{h}} & =\frac{1}{3} \mu^{2}\left[1-\frac{1}{4}\left(1-3 \cos ^{2} \beta\right) S\right] .
\end{aligned}
$$

According to Eqs. (5) and (6), in the cholesteric phase the liquid crystal permittivity tensor $\varepsilon_{i j}^{\mathrm{h}}$ becomes

$$
\varepsilon_{\|}^{h}=1+\frac{N h F}{\varepsilon_{0}}\left\{\bar{\alpha}-\frac{1}{3} \Delta \alpha S+F \frac{\mu^{2}}{3 k T}\left[1+\frac{1}{2}\left(1-3 \cos ^{2} \beta\right) S\right]\right\},
$$




$$
\varepsilon_{\perp}^{\mathrm{h}}=1+\frac{N h F}{\varepsilon_{0}}\left\{\bar{\alpha}+\frac{1}{6} \Delta \alpha S+F \frac{\mu^{2}}{3 k T}\left[1-\frac{1}{4}\left(1-3 \cos ^{2} \beta\right) S\right]\right\} .
$$

The dielectric anisotropy of a cholesteric, defined as $\Delta \varepsilon^{\mathrm{h}}=\varepsilon_{\|}^{\mathrm{h}}-\varepsilon_{\perp}^{\mathrm{h}}$ can be expressed as

$$
\Delta \varepsilon^{h}=\frac{N h F}{\varepsilon_{0}}\left[-\frac{1}{2} \Delta \alpha S+F \frac{\mu^{2}}{4 k T}\left(1-3 \cos ^{2} \beta\right) S\right]
$$

In the isotropic phase (similarly as for the nematics), when $S=0$ and additionally,

$$
\varepsilon_{\perp}^{\mathrm{h}}=\varepsilon_{\|}^{\mathrm{h}}=\varepsilon, \quad \varepsilon_{\|}=\varepsilon_{\perp}=\varepsilon,
$$

Eqs. (7) and (8) would reduce to the Onsager formula [3]:

$$
\varepsilon=\frac{N h F}{\varepsilon_{0}}\left(\alpha+F \frac{\mu^{2}}{3 k T}\right) .
$$

\section{Results and discussion}

Optical birefringence $(\Delta n)$ measurements have been made for two materials D4 and CE8 in the cholesteric phase. The results are displayed in Fig. 1.
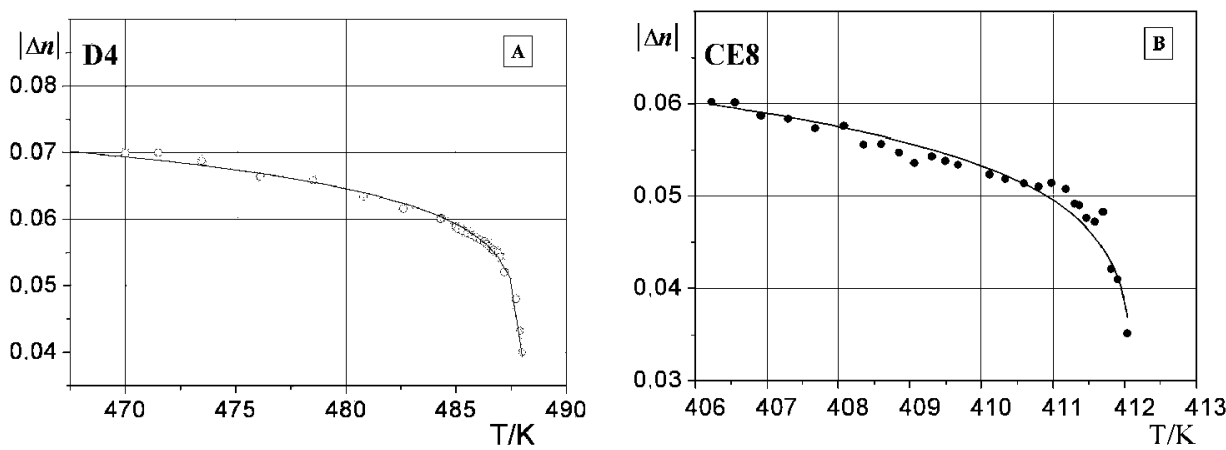

Fig. 1. Optical birefringence $\Delta n$ measured with the modified wedge method (the lens of $14 \mathrm{~mm}$ diameter and curvature radius $R=21.7$ ). (A), (B) D4 and CE8 data, respectively.

The electric permittivity of the two materials D4 and CE8 was measured in two configurations as a function of temperature. The results are shown in Fig. 2.

The method, described by Kuczyński et al. [7], offers the possibility to determine the temperature dependence of $S$ from the optical birefringence $\Delta n$. According to [7] one may write

$$
\Delta n=\Delta n_{0}\left(1-\frac{T}{T^{*}}\right)^{\gamma}
$$



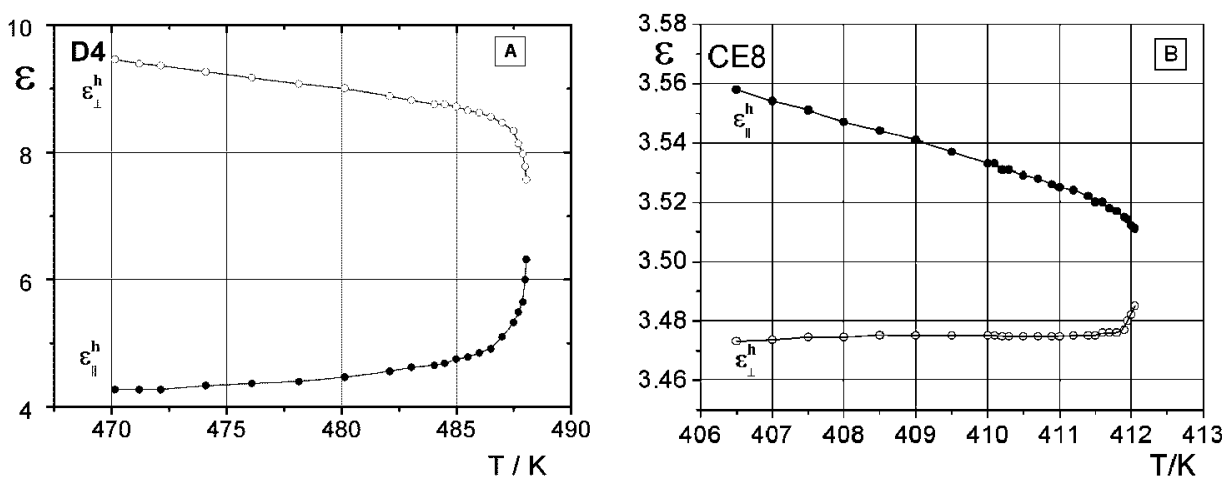

Fig. 2. Electric permittivity; (A), (B) D4 and CE8 data, respectively. The solid lines are drawn following the calculations based on Eqs. (7) and (8) with the parameters of Table.

where $\Delta n$ - optical birefringence of cholesteric liquid crystal, $\Delta n_{0}$ - the assumed magnitude of $\Delta n_{0}$ extrapolated to $0 \mathrm{~K}$ temperature, $T^{*}$ and $\gamma$ are constants $\left(T^{*}\right.$ is about $1-4 \mathrm{~K}$ higher than clearing temperature and the exponent $\gamma<1$ ) [7]. The order parameter $S$ is described with the formula

$$
S=\frac{\Delta n}{\Delta n_{0}}
$$

With the standard numerical fit of $\Delta n$ as a function of temperature the parameters $\Delta n_{0}, T$, and $\gamma$ were determined. Subsequently, entering these values into Eq. (11) the results $S=f(T)$ were displayed as the solid lines in Fig. 3. The order of magnitude of $S$ in the investigated temperature range is characteristic of the nematics.
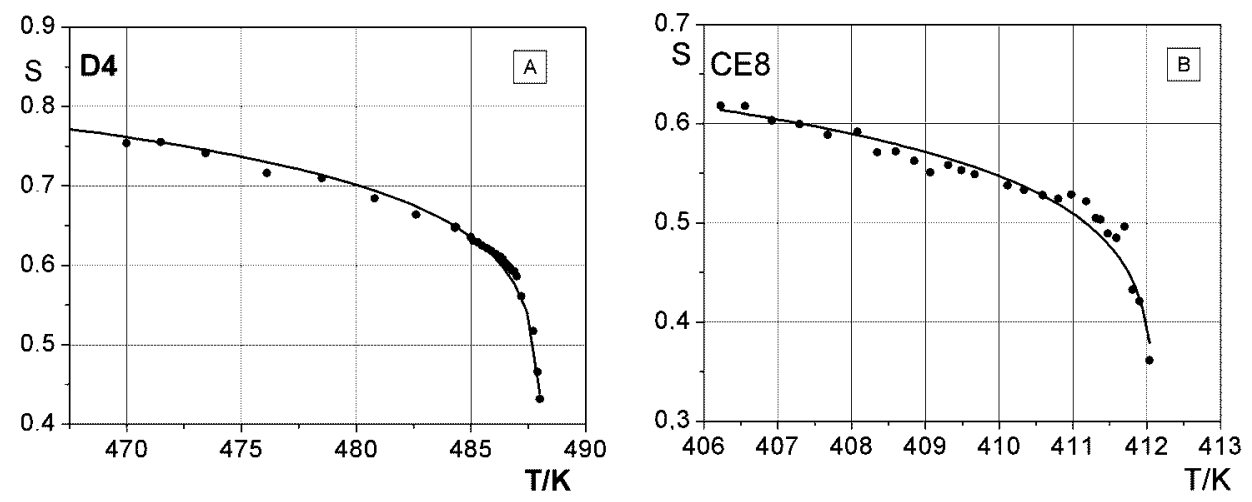

Fig. 3. Temperature dependence of the order parameter $S$; (A), (B) D4 and CE8 data, respectively. The results $S=f(T)$ calculated from Eq. (11) were displayed as the solid lines. 
The experimental values of the temperature dependence of electric permittivity (the experimental points in Figs. $2 \mathrm{~A}$ and $2 \mathrm{~B}$ ) have been used for the determination of $\alpha, \mu$, and $\beta$ of the samples.

The numerical fit of these data sets was based on Eqs. (7) and (8) and the dependence $S=f(T)$ available from the measurements of the birefringence. The final results of the calculations and data calculated using the semi-empirical MP3 method by Małecki [8] are collected in Table. Each value of the parameters

\section{TABLE}

Molecular parameters of the investigated liquid crystals.

\begin{tabular}{l|c|c|c|c}
\hline \hline \multirow{2}{*}{ Parameter } & \multicolumn{2}{|c|}{$\mathrm{D} 4$} & \multicolumn{2}{c}{ CE8 } \\
\cline { 2 - 5 } & this work & {$[8]$} & this work & {$[8]$} \\
\hline$\alpha\left[10^{-40} \mathrm{~F} \mathrm{~m}^{2}\right]$ & $37 \pm 4$ & 39 & $55 \pm 5$ & 46 \\
$\Delta \alpha\left[10^{-40} \mathrm{~F} \mathrm{~m}^{2}\right]$ & $42 \pm 4$ & 46 & $35 \pm 4$ & 40 \\
$\mu\left[10^{-30} \mathrm{C} \mathrm{m}\right]$ & $19 \pm 1$ & 20 & $6 \pm 1$ & 7 \\
$\beta\left[^{\circ}\right]$ & $23 \pm 5$ & 32 & $70 \pm 5$ & 65
\end{tabular}

$\alpha, \mu$, and $\beta$ is an average of the set of numbers measured in the experiment. The accuracy of the parameter magnitude is defined as the largest deflection from the mean value. When discussing the results gathered in Table one finds that the predicted values agree within $10 \%$ with those obtained from the best fit to the experimental data.

Figure 2 presents the argument corroborating the correctness of the numbers determined above. Together with the experimental data points the solid lines are drawn following the calculations based on the formulas (7) and (8) with the parameters of Table. The discrepancy between the experiment and the theory is within a few percent.

With regard to the anisotropy of dielectric properties of the cholesteric liquid crystals one ought to take into account Eqs. (9). Since $N, h, F, \varepsilon_{0}, \Delta \alpha, S$, and $\mu^{2}$ are positive, it follows from Eq. (9), the sign of the anisotropy of cholesterics is determined by the expression

$$
A=-\frac{1}{2} \Delta \alpha S+F \frac{\mu^{2}}{4 k T}\left(1-3 \cos ^{2} \beta\right) S .
$$

The only term of Eq. (12), which may change sign, are the bracket $\left(1-3 \cos ^{2} \beta\right)$. It will be negative for the angles $\beta$ between 0 and $54.74^{\circ}$ which means that also $\Delta \varepsilon^{\mathrm{h}}<0$. For the angles $\beta$ from the range $54.74^{\circ}<\beta<90^{\circ}$ the expression $1-3 \cos ^{2} \beta$ is positive and the sign of $\Delta \varepsilon^{\mathrm{h}}$ will depend on the inequality

$$
F \frac{\mu^{2}}{3 k T}\left(1-3 \cos ^{2} \beta\right)>\frac{1}{2} \Delta \alpha
$$

Contrary to the properties of nematics, this means that the condition $\Delta \varepsilon^{\mathrm{h}}>0$ 

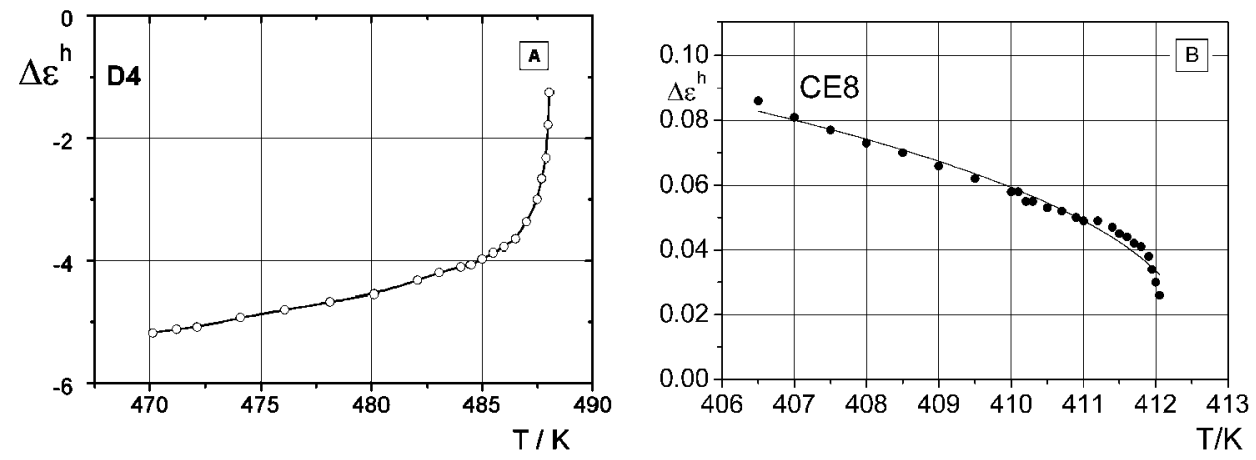

Fig. 4. Dielectric anisotropy $\left(\Delta \varepsilon^{\mathrm{h}}=\varepsilon_{\|}^{\mathrm{h}}-\varepsilon_{\perp}^{\mathrm{h}}\right)$ of cholesteric liquid crystals (A) D4 and (B) CE8 data.

can only be observed in the liquid crystals consisting of molecules whose dipole moment is transversal to the "long axis direction" $\left(54.74^{\circ}<\beta<90^{\circ}\right)$ and in the situations where the orientational part of the dielectric polarization is larger than the contribution due to the anisotropy of the polarizability $\Delta \alpha$.

When

$$
F \frac{\mu^{2}}{4 k T}\left(1-3 \cos ^{2} \beta\right)=\frac{1}{2} \Delta \alpha
$$

it follows from Eq. (9) that $\Delta \varepsilon^{\mathrm{h}}=0$, therefore, in static electric fields the cholesteric liquid crystal will act as an isotropic dielectric. All the above listed equations adequately describe the experimental results. Figure 4 displays the temperature dependence of $\Delta \varepsilon^{\mathrm{h}}$ for the two materials. In the material coded as D4 the dipole moment makes with the "long axis direction" an angle $\beta \approx 30^{\circ}<54.74^{\circ}, \Delta \varepsilon^{\mathrm{h}}<0$ (Fig. 4A). On the other hand, for the liquid crystal CE $8, \beta \approx 70^{\circ}>54.74^{\circ}$ and $\Delta \varepsilon^{\mathrm{h}}>0$ (Fig. 4B)

\section{Conclusions}

The model presented here relates the electric permittivity values $\varepsilon_{\|}^{\mathrm{h}}$, $\varepsilon_{\perp}^{\mathrm{h}}$ of the macroscopic samples of the cholesteric liquid crystals to the actual molecular parameters $(\alpha, \mu, \beta)$. It opens a way for the calculation of the average polarizability, but also it makes possible to find its anisotropy $\Delta \alpha$ and the principal components $\alpha_{\|}$and $\alpha_{\perp}$ of the tensor $\alpha_{i j}$.

The precision of the method can be improved by the application of an independent measurement of optical refractive indices. The previously pronounced relation [6], between the dielectric anisotropy of the cholesteric liquid crystals $\Delta \varepsilon^{\mathrm{h}}$ and the dielectric anisotropy $\Delta \varepsilon$ of the nematics made of identical molecules, can further be corroborated with the arguments based on Eqs. (7)-(9). It becomes possible to predict the sign of $\Delta \varepsilon^{\mathrm{h}}$ from the magnitude of $\beta$ - the angle between 
the long axis of molecule and the dipole moment. Despite the necessary simplifying assumptions the results accomplished with this model could be appreciated.

\section{Acknowledgments}

The author thanks Professor Jerzy Małecki and Professor Wojciech Kuczyński for very valuable remarks. This work was supported by the State Committee for Scientific Research under project 2PO3B 12722.

\section{References}

[1] P.G. de Gennes, Physics of Liquid Crystals, Clarendon Press, Oxford 1974.

[2] W. Maier, G. Meier, Z. Naturforsch. A 16, 262 (1961).

[3] L. Onsager, J. Am. Chem. Soc. 58, 1486 (1936).

[4] M.F. Vuks, Opt. Spektrosk. 20, 361 (1966).

[5] J. Jadżyn, S. Czerkas, G. Czechowski, A. Burczyk, R. Dąbrowski, Liq. Cryst. 26, 437 (1999).

[6] J. Hoffmann, Acta Phys. Pol. A 103, 41 (2003).

[7] W. Kuczyński, B. Żywucki, J. Małecki, Mol. Cryst. Liq. Cryst. 381, 1 (2002).

[8] J. Małecki, unpublished results. 